\title{
PREVALENCIA Y ACTITUDES HACIA EL USO DEL TABACO EN ESTUDIANTES DE TERAPIA RESPIRATORIA
}

Amparo Elizabeth Cristancho Montenegro ${ }^{1}$, Gloria Esperanza Mora Castiblanco ${ }^{1}$, Dario Mendoza Romero ${ }^{1}$

\section{Resumen}

Introducción: Esta investigación realiza una valoración de la prevalencia del uso de tabaco y actitudes relacionadas en estudiantes de terapia respiratoria. Métodos. Un diseño observacional descriptivo transversal fue utilizado con 127 estudiantes. La encuesta mundial de tabaquismo en estudiantes de profesiones de la salud (GHPSS) fue empleada en este estudio. Resultados. 20,47\% de la muestra informó uso del cigarrillo en el último mes y 53,54\% manifestó haberlo hecho en el último año. Las mujeres presentaron mayor consumo que los hombres $(\mathrm{p}=0,027) .60 \%$ de la muestra presenta exposición al humo de cigarrillo de segunda mano y $44 \%$ informó haber probado el cigarrillo antes de los 19 años. Conclusion. La prevalencia de tabaco en el último mes fue superior a la observada en Colombia. Se hace necesario reforzar las medidas de ambientes libres de tabaco en universidades e intervenir en estudiantes de terapia respiratoria con técnicas para la cesación del uso de tabaco, así como promover hábitos saludables.

${ }^{1}$ Grupo de investigación Aire Libre, Fundación Universitaria del Área Andina, Bogotá, Colombia. 


\section{PREVALENCE AND ATTITUDES TOWARDS THE USE OF TOBACCO IN STUDENTS OF RESPIRATORY THERAPY}

Amparo Elizabeth Cristancho Montenegro ${ }^{1}$, Gloria Esperanza Mora Castiblan$\mathrm{Co}^{1}$, Dario Mendoza Romero ${ }^{1}$

\section{Abstract}

Introduction. This research assesses the prevalence of the use of tobacco and related attitudes in respiratory therapy students. Methods. A transversal descriptive and observational study was carried out with 127 subjects. The global survey of smoking among students of health programs (GHPSS) was applied in this study. Results. $20.47 \%$ of the sample reported cigarette consumption in the last month and $53.54 \%$ reported have done it in the last year. Women showed higher consumption than men $(\mathrm{p}=0.027) .60 \%$ of the sample subjects said they were passively exposed to cigarette smoke. and $44 \%$ reported having tried cigarettes before they were 19 years. Conclusion. The prevalence of tobacco in the last month was higher than that the observed in Colombia. It is necessary to reinforce measures of smoke-free environments in universities and make an intervention with respiratory therapy students with techniques to cease the tobacco consumption, as well as promoting healthy habits. 


\section{PREVALENCE AND ATTITUDES TOWARDS THE USE OF TOBACCO IN STUDENTS OF RESPIRATORY THERAPY}

Amparo Elizabeth Cristancho Montenegro ${ }^{1}$, Gloria Esperanza Mora Castiblan$\mathrm{Co}^{1}$, Dario Mendoza Romero ${ }^{1}$

\section{Resumo}

Introdução. Esta investigação faz uma avaliação da prevalência do uso do tabaco e as atitudes relacionadas em estudantes de terapia respiratória. Métodos. Utilizou-se um desenho observacional descritivo transversal com 127 alunos. A pesquisa global sobre tabagismo entre estudantes universitários da área da saúde (Global Health Professional Students Survey, GHPSS) foi utilizada neste estudo. Resultados. $20,47 \%$ da amostra relataram uso de cigarro no último mês e $53,54 \%$ manifestaram fazer isso no ano passado. As mulheres apresentaram maior consumo do que os homens ( $\mathrm{p}=0,027) .60 \%$ da amostra apresenta exposição à fumaça de cigarro de segunda mão e $44 \%$ relataram ter experimentado com o cigarro antes dos 19 anos. Conclusão. A prevalência do tabaco no último mês foi superior à observada na Colômbia. É necessário reforçar as medidas de ambientes livres de tabaco em universidades e intervir em estudantes de terapia respiratória com técnicas para cessação do uso do tabaco, bem como promover hábitos saudáveis. 


\section{Introducción}

El tabaco es la principal causa de muerte prevenible en el mundo; cerca de seis millones de personas mueren en el mundo por causa de sus efectos cada año. Más de cinco millones de esas muertes son el resultado del uso directo del tabaco y más de 600000 son muertes generadas por el humo de segunda mano (1). Sin embargo, muchas de esas muertes y de los efectos malignos del tabaco pueden ser prevenibles en un $90 \%$, si la población menor de 30 años logra abandonar el hábito de fumar (2).

En Colombia, si bien la prevalencia de haber fumado tabaco en el último mes mostró reducción entre el 1993 y el 2007 ( $21,4 \%$ en $1993,18,9 \%$ en $1999,17 \%$ en 2003 y 12,7\% en 2007) (3), según datos obtenidos de la Encuesta Nacional de Consumo de Sustancias Psicoactivas(4), en 2008 aumentó un 17,06\% y disminuyó un $12,95 \%$ en 2013 . Este panorama evidencia que desde el 2007 no se ha logrado disminuir el porcentaje de fumadores en el país y que más de 3 millones de personas en Colombia fuman cigarillo. La ciudad con mayor frecuencia de consumo de tabaco es Medellín y su área metropolitana, con un 19,5\%, seguida de Bogotá con un 16,89\% (4).

Diferentes estudios se han centrado en los fenómenos que ocurren en estudiantes de ciencias de la salud con el uso del cigarrillo (5-7), en los que se aprecia como factor común la falta de preparación adecuada para enfrentar esta problemática en la sociedad $y$, en algunos casos, entre sus colegas. Se espera que los estudiantes de terapia respiratoria sean ejemplo de estilos de vida saludable y que brinden soporte a sus futuros pacientes en ambientes libres de humo, de manera similar a lo que se espera de otros profesionales de la salud (8). Sin embargo, se desconoce la prevalencia, grado de uso y comportamientos asociados al uso de tabaco en estos estudiantes.

Los terapeutas respiratorios, así como los demás profesionales de la salud, juegan un papel fundamental en asumir un rol positivo y ser ejemplo de comportamientos saludables, a fin de facilitar la disminución del uso del tabaco en la población. Para esto, los estudiantes deben primero incorporar estos comportamientos en sus estilos de vida $(9,10)$. El objetivo de este estudio fue conocer la prevalencia del consumo de tabaco y las actitudes relacionadas con éste en los estudiantes de terapia respiratoria de una universidad en Bogotá, D.C.

\section{Materiales y Métodos}

\section{Diseño y población de estudio}

Se realizó un estudio observacional de tipo descriptivo transversal que empleó la encuesta mundial de tabaquismo (GHPSS) en estudiantes de profesiones de la salud. El cuestionario se aplicó a estudiantes de terapia respiratoria de una universidad en Bogotá, Colombia. De un total de los 129 estudiantes, 127 estudiantes aceptaron responder el instrumento, lo que corresponde al 98,45\% de la población. Por lo general, este tipo de estudiantes trabaja durante el día, razón por la que se requería en ocasiones pedir autorización a los docentes para que los estudiantes pudiesen contestar 
el cuestionario, previa autorización de este. El programa de terapia respiratoria tiene una duración de 8 semestres (4 años) y se registraron datos de todos los semestres.

\section{Instrumento}

La GHPSS es un instrumento desarrollado por la Organización Mundial de la Salud (OMS), el Centro para el Control y la Prevención de Enfermedades de los Estados Unidos (CDC) y la Asociación Canadiense de Salud Pública (9) para obtener datos sobre el uso del tabaco y realizar asesorías acerca de su suspensión entre estudiantes de profesiones de la salud. Inicialmente, fue utilizado en estudiantes de medicina, enfermería, odontología y farmacia. La GHPSS hace parte del sistema mundial de vigilancia del tabaco e indaga sobre variables demográficas, la prevalencia del consumo de cigarrillo y otros usos del tabaco, los conocimientos y actitudes sobre uso del tabaco, la exposición al humo de segunda mano, el deseo de dejar de fumar y el entrenamiento recibido para asesorar al paciente en técnicas para abandonar el tabaco (9).

Aunque la GHPSS se ha utilizado con estudiantes de otras profesiones en estudios previos, su diseño está orientado específicamente hacia estudiantes de profesiones de la salud $(7,8,11,12)$. Es deseable que los terapeutas respiratorios ofrezcan consejo y sirvan de ejemplo para ambientes libres de humo en sus futuros pacientes, ya que se entiende que su principal acción promocional es en salud cardiorrespiratoria.
Para el presente estudio se adaptó la versión de la GHPSS empleada en contextos latinoamericanos (10). La adaptación se realizó con un grupo de 20 estudiantes para asegurar el adecuado entendimiento de cada pregunta y se capacitaron cuatro personas para administrar el cuestionario en forma personal.

\section{Obtención y manejo de datos}

La captura de datos se realizó mediante la aplicación Epi Info 7 (versión 7.1.5) para Windows y se exportó el cuestionario a la versión Epi Info Android, de manera que cada encuestador capacitado administró la GHPSS desde su teléfono celular. Este proceso permitió controlar el progreso de los encuestadores, asegurar el entendimiento de cada pregunta por parte del entrevistado y contribuir de forma responsable con el ambiente, al no emplear formularios en papel.

Una vez recogida la información de los dispositivos móviles (teléfonos celulares con la aplicación), los datos se transfirieron a un computador de escritorio con la versión para Windows de Epi Info 7. A su vez, se exportaron los datos al programa estadístico IBM SPSS versión 23, donde se etiquetaron todas las variables de la GHPSS. Los datos se presentan en forma de frecuencias y porcentajes de un total de 127 estudiantes. Las comparaciones de frecuencias se realizaron con la prueba chi-cuadrado.

\section{Consideraciones éticas}

Este estudio fue aprobado por el comité de investigaciones de la Fundación Universitaria del Área Andina. La participa- 
ción de los estudiantes de terapia respiratoria fue voluntaria y, de acuerdo con lo establecido en la resolución 8430 de 1993 del Ministerio de Salud de Colombia, esta se consideró una investigación de riesgo mínimo por tratarse de personal subordinado. La investigación cumplió todas las normas éticas establecidas en la Declaración de Helsinki y se preservó la confidencialidad de la información.

\section{Resultados}

Los cuestionarios fueron respondidos por $113(88,97 \%)$ mujeres y $14(11,02 \%)$ hombres. La edad promedio en las mujeres fue 22,94 años (D.E. 3,24 años) y la de los hombres 22,21 años (D.E. 3,26 años).

Tabla 1. Prevalencia del uso del tabaco entre estudiantes de terapia respiratoria de una universidad de Bogotá, D.C. $(n=127)$.

\begin{tabular}{l|l|l|l|l}
\hline & $\begin{array}{c}\text { Cigarrillo en } \\
\text { curso de vida }\end{array}$ & $\begin{array}{c}\text { Actuales } \\
\text { fumadores }\end{array}$ & $\begin{array}{c}\text { Otros productos } \\
\text { distintos al cigarrillo } \\
\text { en la vida }\end{array}$ & $\begin{array}{c}\text { Actuales fumadores } \\
\text { de otros productos } \\
\text { distinto al cigarrillo }\end{array}$ \\
\hline Mujer & $59(46,45 \%)$ & $20(15,75 \%)$ & $8(6,3 \%)$ & $6(4,72 \%)$ \\
Hombre & $9(7,08 \%)$ & $6(4,72 \%)$ & $2(1,57 \%)$ & $2(1,57 \%)$ \\
Total & $68(53,54 \%)$ & $26(20,47 \%)$ & $10(7,87 \%)$ & $8(6,3 \%)$ \\
valor p & 0,3928 & 0,027 & 0,345 & 0,192 \\
\hline
\end{tabular}

Los porcentajes son mostrados con respecto al total de la muestra $(n=127)$.

\section{Prevalencia y edad de inicio del consumo}

Los datos de prevalencia de cigarrillo en el curso de la vida y de fumadores actuales se presentan en la Tabla 1. El 20,47\% de los estudiantes son fumadores actua- les, de los cuales un $15,75 \%$ eran mujeres y un $4,72 \%$ hombres $(p=0,027)$. Con respecto a la edad de inicio del consumo de tabaco, un 15,7\% informó que fue entre 11 y 15 años; otro $15,7 \%$ fue entre 16 y 17 años y un 13,4\% entre 18 y 19 años (ver Tabla 2). 
Tabla 2. Edad de inicio de fumar y uso de cigarrillo y otros productos en estudiantes de terapia respiratoria

\begin{tabular}{|c|c|c|c|}
\hline & Mujer & Hombre & Total \\
\hline $\begin{array}{l}\text { Edad de inicio de fumar } \\
\text { (años) }\end{array}$ & n (\%) & n (\%) & n (\%) \\
\hline 11 a 15 & $14(11,0 \%)$ & $6(4,7 \%)$ & $20(15,7 \%)$ \\
\hline 16 a 17 & $19(15,0 \%)$ & $1(0,8 \%)$ & $20(15,7 \%)$ \\
\hline 18 a 19 & $16(12,6 \%)$ & $1(0,8 \%)$ & $17(13,4 \%)$ \\
\hline 20 a 24 & $9(7,1 \%)$ & 0 & $9(7,1 \%)$ \\
\hline 25 a 29 & $1(0,8 \%)$ & 0 & $1(0,8 \%)$ \\
\hline 30 o más & 0 & $1(0,8 \%)$ & $1(0,8 \%)$ \\
\hline $\begin{array}{l}\text { Si fuman cigarrillos en } \\
\text { recintos de la Facultad }\end{array}$ & $14(11 \%)$ & $4(3,1 \%)$ & $18(14,2 \%)$ \\
\hline $\begin{array}{l}\text { Si fuman cigarrillos al } \\
\text { interior de la Facultad }\end{array}$ & $6(4,7 \%)$ & $3(2,4 \%)$ & $9(7,1 \%)$ \\
\hline $\begin{array}{l}\text { Si fuman otros productos } \\
\text { al interior de la Facultad }\end{array}$ & $7(5,5 \%)$ & $1(0,8 \%)$ & $8(6,3 \%)$ \\
\hline
\end{tabular}

Los porcentajes son mostrados con respecto al total de la muestra $(\mathrm{n}=127)$.

Tabla 3. Variables relacionadas con la exposición al humo de cigarrillo, venta y publicidad.

\begin{tabular}{lcccc}
\hline & Mujer & Hombre & Total \\
\hline $\begin{array}{l}\text { Exposición cigarrillo lugar } \\
\text { de vivienda }\end{array}$ & $\mathrm{n}(\%)$ & $\mathrm{n}(\%)$ & $\mathrm{n}(\%)$ \\
Ninguno & $66(52,0 \%)$ & $3(2,4 \%)$ & $69(54,3 \%)$ \\
1 a 2 días & $19(15,0 \%)$ & $6(4,7 \%)$ & $25(19,7 \%)$ \\
3 a 4 días & $12(9,4 \%)$ & $0(0,0 \%)$ & $12(9,4 \%)$ \\
5 a 6 días & $4(3,1 \%)$ & $1(0,8 \%)$ & $5(3,9 \%)$ \\
Todos los días & $12(9,4 \%)$ & $4(3,1 \%)$ & $16(12,6 \%)$ \\
\hline
\end{tabular}


INVESTIGACIONES ANDINA No. 37, Vol. 20

Continuación Tabla 3. Variables relacionadas con la exposición al humo de cigarrillo, venta y publicidad.

\begin{tabular}{|c|c|c|c|}
\hline & Mujer & Hombre & Total \\
\hline \multicolumn{4}{|l|}{$\begin{array}{l}\text { Exposición cigarrillo } \\
\text { lugares distintos a vivienda }\end{array}$} \\
\hline Ninguno & $48(37,8 \%)$ & $3(2,4 \%)$ & $51(40,2 \%)$ \\
\hline 1 a 2 días & $29(22,8 \%)$ & $3(2,4 \%)$ & $32(25,2 \%)$ \\
\hline 3 a 4 días & $15(11,8 \%)$ & $3(2,4 \%)$ & $18(14,2 \%)$ \\
\hline 5 a 6 días & $8(6,3 \%)$ & $1(0,8 \%)$ & $9(7,1 \%)$ \\
\hline Todos los días & $13(10,2 \%)$ & $4(3,1 \%)$ & $17(13,4 \%)$ \\
\hline $\begin{array}{l}\text { Existen lugares para fumar } \\
\text { en la Facultad (sí) }\end{array}$ & $22(17,3 \%)$ & $4(3,1 \%)$ & $26(20,5 \%)$ \\
\hline \multicolumn{4}{|l|}{$\begin{array}{l}\text { Existen programas de } \\
\text { ayuda en la Universidad } \\
\text { para dejar de fumar }\end{array}$} \\
\hline Sí & $22(17,5 \%)$ & $1(0,8 \%)$ & $23(18,3 \%)$ \\
\hline No & $12(9,5 \%)$ & $1(0,8 \%)$ & $13(10,3 \%)$ \\
\hline No se & $78(61,9 \%)$ & $12(9,5 \%)$ & $90(71,4 \%)$ \\
\hline $\begin{array}{l}\text { Prohibición venta de } \\
\text { tabaco a menores (sí) }\end{array}$ & $101(79,5 \%)$ & $13(10,2 \%)$ & $114(89,8 \%)$ \\
\hline $\begin{array}{l}\text { Prohibición publicidad } \\
\text { tabaco (Sí) }\end{array}$ & $89(70,1 \%)$ & $7(5,5 \%)$ & $96(75,6 \%)$ \\
\hline \multicolumn{4}{|l|}{$\begin{array}{l}\text { Humo de cigarrillo de } \\
\text { otros, ¿es dañino? }\end{array}$} \\
\hline Absolutamente no & $4(3,1 \%)$ & $1(0,8 \%)$ & $5(3,9 \%)$ \\
\hline Probablemente no & $18(14,2 \%)$ & $0(0,0 \%)$ & $18(14,2 \%)$ \\
\hline Probablemente sí & $13(10,2 \%)$ & $1(0,8 \%)$ & $14(11,0 \%)$ \\
\hline Absolutamente sí & $78(61,4 \%)$ & $12(9,4 \%)$ & $90(70,9 \%)$ \\
\hline
\end{tabular}

Los porcentajes son mostrados con respecto al total de la muestra $(n=127)$.

\section{Consumo dentro de la universidad}

De los estudiantes que fuman actualmente, un 14,2\% manifestó haberlo hecho en recintos de la universidad, un $7,1 \%$ al interior de edificios de la universidad y un 6,3\% informó acerca del uso de otros productos distintos al cigarrillo 
al interior de la universidad (ver Tabla 2).

\section{Exposición en la vivienda}

Con respecto a la exposición al humo de cigarrillo en el lugar de vivienda, el $54,3 \%$ informó no estar expuesto, mientras que el 45,6\% sí lo está. Ahora bien, si se suman los expuestos al humo de cigarrillo en lugares distintos a los de su vivienda, se aprecia que la mayoría (59,9\%) presentan esta condición. La situación en cuanto al daño que el humo de cigarrillo de otras personas puede ge- nerar en la salud presenta una opinión de absolutamente sí en el 70,9\% de los estudiantes (Tabla 3).

\section{Actitudes hacia el uso del tabaco}

El 90\% de los estudiantes está de acuerdo con la prohibición de la venta de cigarrillos a menores de 18 años y el 75\% con la prohibición de toda la publicidad relacionada con el tabaco. La mayoría de los estudiantes $(71,4 \%)$ desconoce la existencia de programas de ayuda en la universidad para dejar de fumar (ver Tabla 3).

Tabla 4. Variables relacionadas con actitudes frente al cigarrillo, exposición al humo de segunda mano y cesación.

\begin{tabular}{|c|c|c|c|}
\hline & Mujer & Hombre & Total \\
\hline \multicolumn{4}{|l|}{ Sensación cuando alguien fuma } \\
\hline $\begin{array}{l}\text { Nada, no me importa, me da lo } \\
\text { mismo }\end{array}$ & $16(12,7 \%)$ & $6(4,8 \%)$ & $22(17,5 \%)$ \\
\hline Me agrada el humo del cigarrillo & $10(7,9 \%)$ & $1(0,8 \%)$ & $11(8,7 \%)$ \\
\hline $\begin{array}{l}\text { En algunas ocasiones me siento } \\
\text { molesto (a) }\end{array}$ & $15(11,9 \%)$ & $3(2,4 \%)$ & $18(14,3 \%)$ \\
\hline Siempre me siento molesto (a) & $66(52,4 \%)$ & $2(1,6 \%)$ & $68(54,0 \%)$ \\
\hline Otro & $5(4,0 \%)$ & $2(1,6 \%)$ & $7(5,6 \%)$ \\
\hline $\begin{array}{l}\text { Alguna vez ha pedido que no } \\
\text { fumen en su presencia (sí) }\end{array}$ & $81(63,0 \%)$ & $5(3,9 \%)$ & $86(66,9 \%)$ \\
\hline $\begin{array}{l}\text { ¿Profesionales de la salud deben } \\
\text { recibir entrenamiento sobre } \\
\text { dejar de fumar? (sí) }\end{array}$ & $101(79,5 \%)$ & $13(10,2 \%)$ & $114(89,8 \%)$ \\
\hline $\begin{array}{l}\text { Profesionales de la salud son } \\
\text { modelos (sí) }\end{array}$ & $86(67,7 \%)$ & $9(7,1 \%)$ & $95(74,8 \%)$ \\
\hline $\begin{array}{l}\text { Ha intentado dejar de fumar en } \\
\text { el último año (sí) }\end{array}$ & $15(11,9 \%)$ & $6(4,8 \%)$ & $21(16,7 \%)$ \\
\hline Quiere dejar de fumar ahora (sí) & $11(8,7 \%)$ & $6(4,8 \%)$ & $17(13,5 \%)$ \\
\hline $\begin{array}{l}\text { Profesionales que fuman acon- } \\
\text { sejan menos dejar de fumar (sí) }\end{array}$ & $79(62,2 \%)$ & $8(6,3 \%)$ & $87(68,5 \%)$ \\
\hline
\end{tabular}


Uno de los porcentajes más altos de opinión (90\%) de los estudiantes se refiere a la necesidad de recibir entrenamiento por parte de los profesionales sobre técnicas para dejar de fumar. Un acuerdo mayoritario se manifiesta en el sentido que aquellos profesionales que fuman aconsejan dejar este hábito a sus pacientes con menor frecuencia $(68,5 \%)$ (ver Tabla 4).

Frente a actitudes del humo de cigarrillo expulsado por otras personas, $54 \%$ se siente molesto y al $17,5 \%$ no le importa. Dentro de aquellos que manifiestan molestia, la mayoría han actuado y solicitan que no se fume en su presencia (67\%). Tres cuartos de los encuestados consideran que los profesionales de la salud son ejemplos para sus pacientes y la comunidad (Tabla 4).

\section{Discusión de resultados}

La prevalencia de fumadores en el último mes encontrada en estudiantes de terapia respiratoria de una universidad en Bogotá, Colombia, fue de un $20,47 \%$, mayor a la reportada en el último estudio de sustancias psicoactivas en Colombia (4), aunque similar a la observada en estudiantes de medicina de Santa Marta, Colombia (19\%) (11). Esta proporción se puede comparar con la registrada en 1993 en Colombia, cuando superó $20 \%$ (3). Por otro lado, es menor a la encontrada en estudiantes universitarios de Murcia, España (33\%) (12) y en Bogotá, Colombia, en otra universidad con estudiantes de diversas áreas del conocimiento (31\%) (13), aunque esta última encuesta indagó acerca de la pre- valencia de fumar en diversos contextos y lugares, más no durante el último mes. La frecuencia de tabaquismo en el último mes fue significativamente menor en hombres que en mujeres $(\mathrm{p}<0,05)$, aunque esto, posiblemente sea por la reducida proporción de hombres que estudian terapia respiratoria $(11,02 \%)$. Tal hecho puede explicar la mayor prevalencia de mujeres fumadoras que contrasta con la mayoría de estudios $(3,4,6,7,14,15)$. No obstante, coincide con un estudio realizado en estudiantes de carreras de la salud de Chile y que empleó el mismo instrumento; independientemente del programa al que pertenecían, las mujeres fumaban más que sus compañeros hombres(10). Es importante, sin embargo, señalar que la prevalencia de hábitos no saludables y factores de riesgo "modificables" para enfermedad cardiovascular ha venido en aumento en mujeres, motivo por el cual es necesario realizar intervenciones específicamente en ellas, dirigidas a la prevención del consumo de tabaco, la cesación del mismo y la promoción de estilos de vida saludable (16).

Es llamativa la temprana edad de inicio del consumo de tabaco: el $44 \%$ manifiesta haber empezado entre los $11 \mathrm{y}$ 19 años, lo que evidencia la susceptibilidad de los menores de edad, a pesar de la existencia de un marco legal que regula este tipo de prácticas en Colombia(17). Esta proporción es preocupante y fácilmente se ubica por encima de los datos del país y de otros contextos en Latinoamérica(18), aunque es menor a la reportada en Chile(10). Esto puede ser explicado por las características socioeconómicas de los estudiantes que 
ingresan a la universidad, quienes en su mayoría provienen de la periferia de la ciudad (3).

En el estudio se encontró que el $60 \%$ de los estudiantes de terapia respiratoria están expuestos al humo de cigarrillo de segunda mano, entre uno a siete días a la semana. Este porcentaje es mayor al observado en estudiantes de odontología en una universidad en India (41\%) (7) y menor al encontrado en estudiantes de la salud en Chile (86,2\%) (10). Por otra parte, aunque la mayoría (71\%) de los estudiantes están convencidos de que el humo de cigarrillo es absolutamente dañino para la salud, lamentablemente, pocos conocen la presencia de programas al interior de la universidad que les brinden apoyo para abandonar este hábito (18,3\%). Estudiar esta relación puede ser importante para influir en el mantenimiento de ambientes libres de humo y para reforzar la aplicación de la legislación vigente en Colombia sobre venta de cigarrillos al por menor, dado que es posible que esta la principal razón de exposición de cigarrillo de segunda mano en universidades.

Al comparar resultados sobre actitudes frente al cigarrillo, en especial en lo concerniente a pedir a otras personas que no fumen en su presencia, el $67 \%$ de nuestros estudiantes lo hace, frente al $71,5 \%$ de estudiantes en Chile (10). En otros contextos y frente al rol modélico, el $84,3 \%$ de los estudiantes de ciencias de la salud en India (7) manifiestan que el profesional de la salud es ejemplo para sus pacientes y el público; en Arabia
Saudita, entre el $83,3 \%$ y el $91,1 \%$ (14); en el presente estudio, un $74,8 \%$, mientras que en México 77,3\% (18), Uruguay $75,5 \%$ (5) y Chile 68,6\% (10). De lo anterior se desprende que, en América Latina la percepción del rol modélico de los profesionales de la salud es algo más bajo.

Algunas limitaciones del actual estudio se deben al tipo de diseño empleado por el uso del cuestionario, que pueden sobre o infra estimar algunas respuestas dadas por los participantes. Asimismo, puede presentarse algún sesgo de memoria. Sin embargo, la GHPSS ha sido empleada y evaluada en los Estados Unidos (15), mostrando que, al igual que otros cuestionarios sobre tabaco, tiene buena confiabilidad (7).

Se requieren futuras intervenciones para que los estudiantes de terapia respiratoria sean más conscientes de su rol modélico en la prevención y promoción de actitudes frente al tabaco; para que se refuercen las restricciones para la venta al detal y para el consumo de tabaco en instituciones educativas; para que se realicen ajustes al currículo para mejorar las competencias del terapeuta respiratorio, relacionadas con la prevención del uso del tabaco en poblaciones vulnerables; para que se intensifiquen las estrategias de promoción de la salud para mitigar el riesgo de adquirir el hábito de fumar en edades tempranas y para que se difundan programas de cesación del hábito de fumar y la promoción de hábitos y estilos de vida saludable entre la comunidad universitaria. 


\section{Referencias}

1. Organización Mundial de la Salud. [Internet]. Tabaco; 2018 Mar 9 [Citado el año mes día]. Disponible en: http://www. who.int/es/news-room/fact-sheets/detail/ tobacco.

2. Berg C., Schauer G., Rodgers K., Narula S. College student smokers: former versus current and nonsmokers. American Journal of Preventive Medicine. 2012;43(5,s3):229-36.

3. Macias F., Malmusi D., Borrell C. Different patterns by age-group and gender of socioeconomic inequalities in smoking in colombia. Nicotine Tob Res. 2013;15(10):1745-55.

4. Colombia, Ministerio de justicia, Ministerio de Salud y Observatorio de Drogas. Estudio Nacional de Consumo de Sustancias Psicoactivas en Colombia 2013. Bogotá: ALVI Impresores S.A.S.; 2014. 182 p.

5. Llambí L., et al. Conocimientos, creencias, prácticas y actitudes con respecto al consumo de tabaco de estudiantes de Medicina de primer año en Uruguay, 2009. Revista Médica del Uruguay. 2012;28(1):4-12

6. Jradi H., Al-Shehri A. Knowledge about tobacco smoking among medical students in Saudi Arabia: Findings from three medical schools. J Epidemiol Glob Health. 2014;4(4):269-76.

7. Fotedar S., et al. Knowledge of, attitude towards, and prevalence of tobacco use among dental students. Oral Health Dent Manag. 2013;12(2):73-9.

8. Ficarra M., et al. Tobacco use prevalence, knowledge and attitudes among Italian hospital healthcare professionals. Eur J Public Health. 2011;21(1):29-34.

9. The GTSS Collaborative Group. Tobacco use and cessation counselling:
Global Health Professionals Survey Pilot Study, 10 countries, 2005. Tob Control. 2006;15(supl 2):31-4.

10. Burgos A., Zitko P., Guerrero A., Alfaro T.. Encuesta mundial de tabaquismo en estudiantes de profesiones de salud (EMTES). Chile: Ministerio de Salud; 2008. 68 p.

11. Ceballos, G. Del Gordo, R., Campo A. Factores asociados con el consumo diario de cigarrillo en estudiantes de medicina de Santa Marta, Colombia. Rev Facultad Nacional de Salud Pública. 2006;24(1):79-83.

12. Mantilla S., Gómez A., Hidalgo M. Actividad física, tabaquismo y consumo de alcohol, en un grupo de estudiantes universitarios. Revista de Salud Pública [Internet]. 2011 [Citado año mes día];13(5):748-58.

Disponible en: http://www.scielosp.org/ pdf/rsap/v13n5/v13n5a03.pdf

13. Novoa M., Barreto I., Silva M. Consumo de cigarrillo y prácticas culturales en contextos universitarios. Revista Latinoamericana de Psicología [Internet]. 2012 [Citado año mes día]; 2012;44(1):97-110. Disponible en: http://www.scielo.org.co/ pdf/rlps/v44n1/v44n1a10.pdf

14. Al-bedah A., Basahi J., Ahmed SS, Mohamed N. Saudi Arabia Global Health Professional Students Tobacco Survey 2010-2011. Life Science Journal. 2012;9(4):5357-68.

15. Warren C., Jones N., Chauvin J. Tobacco use and cessation counselling: cross-country. Data from the Global Health Professions Student Survey (GHPSS), 2005-7. Tob Control. 2008;17(4):238-47.

16. Shaw LJ, et al. Insights From the NHLBI-Sponsored Women's Ischemia Syndrome Evaluation (WISE) Study. JACC [Internet]. 2006 feb 7 [Citado 
2017 Ene 11];47(3supl):4-20. Disponible en: http://www.onlinejacc.org/ content/47/3_Supplement/S4

17. Colombia, Congreso de la República. Disposiciones por medio de las cuales se previenen daños a la salud de los menores de edad, la población no fumadora y se estipulan políticas públicas para la prevención del consumo del tabaco y el abandono de la dependencia del tabaco del fumador y sus derivados en la población colombiana. Ley 1335. Diario Oifical de la República: 21 de julio de 2009.

18. Reynales L., Vázquez J., Lazcano E. Encuesta Mundial de Tabaquismo en Estudiantes de la Salud, México 2006. Salud pública Méx [Internet]. 2007 [Citado año mes día];49(supl 2):194-204. Disponible en: http://bvs.insp.mx/rsp/articulos/articulo. $p h p ? i d=001997$ 
INVESTIGACIONES ANDINA No. 37, Vol. 20 